13

\title{
Scanning velocity measurement of an acousto-optic deflector*
}

(C) Amir Guessoum

Applied Optics Laboratory, Institute of Optics and Precision Mechanics, Ferhat Abbas University, 19000 Setif , Algérie

Laboratoire d'optique appliqu.e, Institut d'optique et de mécanique de pr.cision, Ferhat Abbas University, 19000 Setif , Algérie

e-mail: Amiroptc@yahoo.fr

Received November 04, 2018

Revised November 04, 2018

Accepted December 07, 2018

Beside a demonstrated theoretical formula that describes the variation of diffracted order angle as a function of time, another formula about the scanning velocity of an acousto-optic deflector is successfully demonstrated with experimental results very close to the theoretical formula. This deflector is obtained using laser beam interaction with frequency modulated ultrasonic sinusoidal wave in water. The particular attention that devoted to select the best experiment method of measuring scanning velocity has enabled us to find promising results; the scanning velocity of each diffracted order varies linearly according to modulating signal frequency as well as frequency excursion and sinusoidally as a function of time. Furthermore, the scanning maximum velocity of the pth diffracted order is $\mathrm{p}$ times the first diffracted order.

DOI: $10.21883 /$ OS.2019.04.47524.318-18

* Полный текст статьи опубликован в английской версии журнала. 